This item was submitted to Loughborough's Research Repository by the author.

Items in Figshare are protected by copyright, with all rights reserved, unless otherwise indicated.

\title{
Prevention of flux decline in electrical microfiltration
}

PLEASE CITE THE PUBLISHED VERSION

PUBLISHER

(C) Taylor \& Francis

VERSION

AM (Accepted Manuscript)

LICENCE

CC BY-NC-ND 4.0

REPOSITORY RECORD

Tarleton, E.S., and Richard J. Wakeman. 2009. "Prevention of Flux Decline in Electrical Microfiltration". figshare. https://hdl.handle.net/2134/5392. 
This item was submitted to Loughborough's Institutional Repository (https://dspace.lboro.ac.uk/) by the author and is made available under the following Creative Commons Licence conditions.

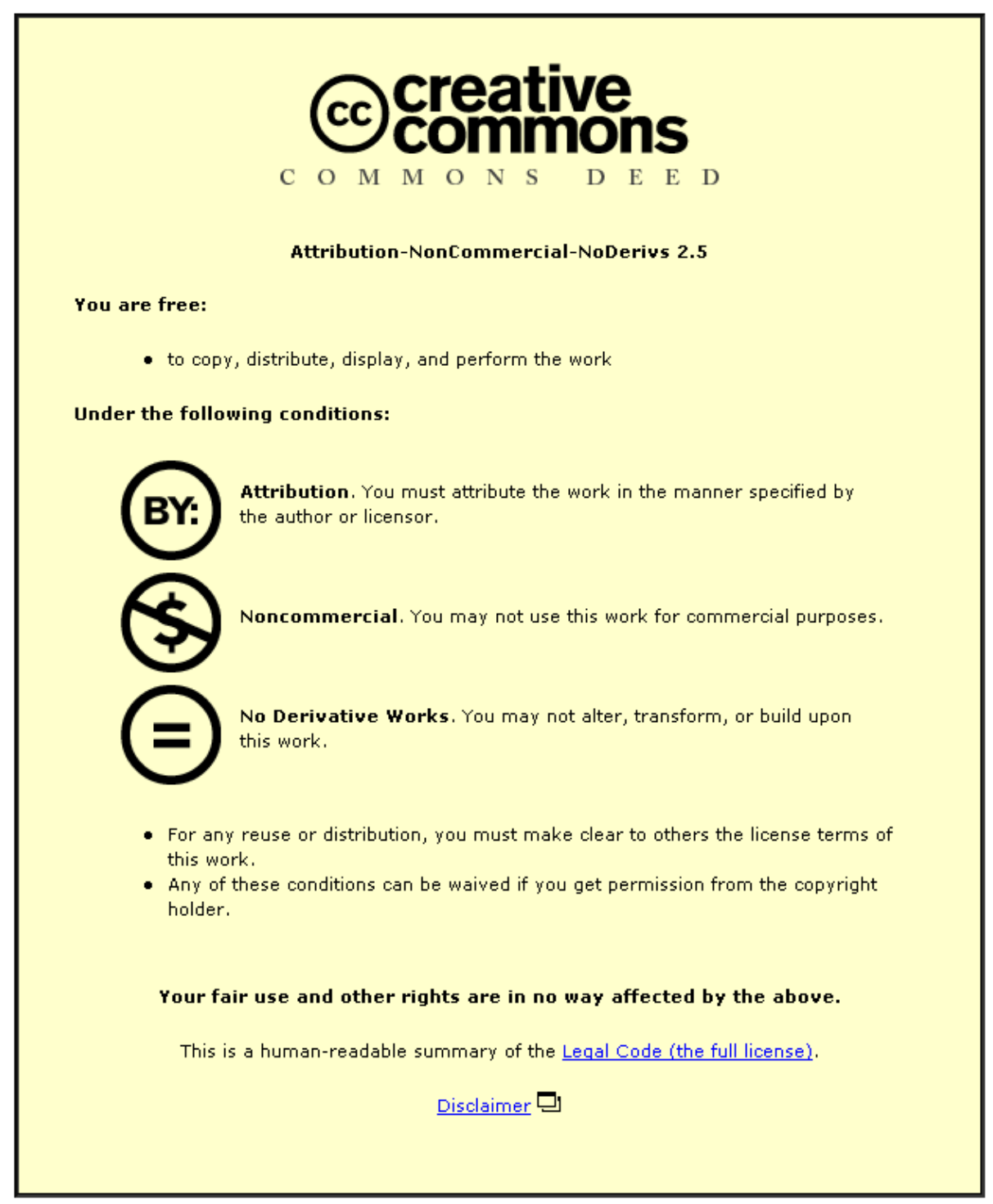

For the full text of this licence, please go to: http://creativecommons.org/licenses/by-nc-nd/2.5/ 


\title{
PREVENTION OF FLUX DECLINE IN ELECTRICAL MICROFILTRATION
}

\author{
E.S. Tarleton (e.s.tarleton@lboro.ac.uk) and R.J. Wakeman \\ Separation Processes Centre, University of Exeter, North Park Road, Exeter, EX4 4QF, UK.
}

\begin{abstract}
Dewatering, or limited thickening, of dilute suspensions can be achieved using microfiltration, but is accompanied by the undesirable permeate flux decline. Experimental data showing this effect are presented, and data indicating some alleviation of the problem using electrical fields are shown. Various models claimed to interpret flux decline are overviewed and discussed. Although some reflect the shape of the flux curve, all must be criticized on the basis that they do not provide any insight as to the causes or the phenomenon.
\end{abstract}

\section{INTRODUCTION}

There is a growing interest in crossflow membrane separation techniques. The development of reverse osmosis (RO), ultrafiltration (UF) and crossflow microfiltration (CFM) has enabled more efficient separation of macromolecular colloidal and near colloidal sized particulates. Such techniques offer the potential advantage of good separation rates with little or no deposit formation at the membrane surface. However, this potential is not generally realised and the permeate flux is seen to decline, thus preventing more widespread use of membrane processes at a large scale. Crossflow filtration differs from conventional deadend filtration in that the bulk flow of suspension is tangential to the filtering surface rather than directly into it. The filter configuration is either of the plate and frame type or tubular. Presently, crossflow filtration systems are employed in many different product applications of solid/liquid separation which include the pharmaceutical, electronics and medical industries, biotechnology, commodity chemicals and water treatments.

Whilst operating commercial units exist the separation of suspensions using crossflow filtration is frequently compromised by membrane fouling (Milisic (1986a)). This can cause a rapid decline in permeate flux during filtration and result in low separation rates. Although the bulk flow of suspension is across the membrane surface, convection carries some particles toward the filter where they can accumulate and lead to the formation of a fouling layer. The mechanisms by which fouling may occur are several, and none are properly and completely understood. The complexity of the interactions between the membrane and the suspension to be filtered have generally hampered attempts to improve crossflow techniques, and represent a real challenge to both experimental and theoretical research. Previous experimental investigations have identified methods by which membrane fouling can potentially be reduced; these include the use of abrasives and filter aids, backwashing/pulsing, pulsed and reversed crossflows, correct membrane choice, careful membrane design, etc. (Bertera et al. (1984); Milisic et al. (1986b)). Recently, methods of assisting filtration by the use of imposed electric, magnetic and sonic fields have been receiving much attention (Henry et al. (1977); Lee et al. (1980); Mikhlin et al. (1982); Muralidhara (1986); Turkson (1987); Dolle (1987)). However, such work is almost entirely experimental and would undoubtedly benefit from a better theoretical understanding.

This paper considers various models which claim to quantify the mechanisms responsible for fouling. Some experimental data are presented which show how CFM can be used to separate fine suspensions effectively, with an emphasis placed on the advantages of using an imposed electric field to assist the filtration. These experimental results are then analysed using both new and existing filtration models to indicate the likely causes of fouling under different processing conditions.

\section{Flux Decline}


The flux decline is caused by several phenomena in, on and near the membrane, sometimes also leading to loss of other advantages such as membrane selectivity. In general the flux decline is caused by a decreased driving force and/or an increased resistance - since flux can be conveniently expressed as:

Flux $=\frac{\text { driving force }(\text { e.g. } \Delta p \text { or } \Delta c)}{(\text { viscosity }) \times(\text { total resistance })}$

The total resistance is the sum of the membrane resistance plus resistances due to, for example, pore blocking, adsorption, gel layer formation, and concentration (and/or particle) polarization. Flux decline phenomena can be divided into fouling processes (considered to be largely irreversible and long term phenomena) and concentration (or particle) polarization (which are mainly reversible and occur rapidly). This paper is mostly concerned with the prevention of particle polarization by the use of electric fields, although the technique probably also has an effect of showing the rate of fouling processes. The terms 'particulate polarization' and 'particulate fouling' will be used synonymously in this paper.

\section{EXPERIMENTAL RESULTS}

The experimental data presented here were obtained using a tubular, pleated crossflow microfilter made from polypropylene or mixed ester cellulose and had a retention rating of $0.2 \mu \mathrm{m}$.

Separation was achieved by pumping aqueous suspension of a known concentration through the annular flow space formed between the vertically mounted membrane cartridge and outer housing. Filtrate was produced within the central core of the cartridge where the flow rate was recorded. The electrodes used to generate the constant voltage DC electric field where positioned so as to traverse the membrane. The experimental data presented here show some typical results obtained from the filter. Many more were obtained which confirm the results shown and these have been presented in previous publications (Wakeman and Tarleton, (1986); (1986a); (1986b); (1987)).

Figure 1 shows the typical effect of increasing the electric field strength on permeate flux for the crossflow filtration of a $0.0039 \% \mathrm{v} / \mathrm{v}$ china clay suspension. When no electric field was applied and the filtration initiated, the permeate flux was found to fall sharply with rapid fouling of the membrane occurring. As the filtration proceeded, the rate at which the flux declined progressively lessened and an equilibrium thickening rate was eventually established in most experiments. If this situation is achieved further particulate fouling of the membrane is negligible and a dynamic equilibrium exists where it is suspected that particles are continually captured and removed from the surface of the membrane deposit at an equal and opposite rate. When the electric field was applied the rate at which the membrane fouled was reduced. The reduced fouling is primarily due to a combination of electrokinetic effects, including electrophoresis which causes less particulates to accumulate within the vicinity of the membrane surface, and electroosmosis within the stationary membrane pores. Figure 2 shows how increasing the electric field strength affects the permeate flux for a more concentrated suspension. With the increased solids content and no electric field, fouling occurred at an initially faster rate: this eventually leading to a very low equilibrium flux. The initial measurable filtration rate was very much lower for the concentrated suspension indicating an extraordinarily rapid rate of particle deposition. The application of the electric field resulted in a considerably improved constant flux which, in this case, was established after about 20 minutes of filtration. These results indicate that membrane fouling can be reduced by using electric fields.

\section{Analysis of the Experimental Results}


Crossflow microfiltration might be regarded as a hybrid operation somewhere between conventional cake filtration and ultrafiltration. As such, CFM might be expected to show agreement with the theories propounded for either UF or cake filtration, or a combination of the two. The analyses presented here use these methods, amongst others, to quantify the effects of fouling in CFM. Comments as to the causes of fouling are largely manifestations of the model developed and the assumptions underpinning that model, and will always be so until irrefutable experiments have been undertaken to verify the causes.

\section{a) Cake Filtration Theory and Microfiltration}

Ideally, crossflow filtration should involve the thickening of a suspension without the formation of a deposit on the membrane. Under such conditions the filtrate flux is likely to be constant and may be predicted by Darcy's law. This may be written as

$$
\frac{d V}{d t}=\frac{A \Delta p}{\mu R_{m}}
$$

where $d V / d t$ is the flow rate under the hydraulic pressure $\Delta p$, of a liquid of viscosity $\mu$ through a filter medium of area $A$ and specific flow resistance $R_{m}$. However, due to a number of factors, particles from the crossflowing suspension are usually found to accumulate within the vicinity of the separating surface and a progressive decline in filtrate flux generally occurs. If sufficient particles are deposited at the filtering surface a 'cake' may form. Under such circumstances it may be more correct to use the batch filtration equation to describe the process.

$$
\frac{d V}{d t}=\frac{A^{2} \Delta p}{\mu\left(\alpha_{a v} c V+A R_{m}\right)}
$$

where $\alpha_{a v}$ is the average specific resistance of the filter cake and $c$ the mass of solid/unit volume filtrate. During CFM some particulate fouling of the membrane is to be expected and it has been reported that classical cake filtration theory can be used as a method of analysis when this occurs (Bertera et al. (1984); Rushton et al.). Shown in Figure 3 is a schematic diagram of the results obtained when equation (2) is applied to a typical set of CFM data. A characteristic of the CFM process is that an equilibrium filtration rate is usually established after an initial fouling period and as such a graph of $d t / d V$ vs. $V$ generally produces a non-linear plot. By design CFM prevents the formation of a true filter cake at the membrane surface, and it is not surprising therefore that the batch filtration equation does not adequately describe the fouling mechanisms which occur. However, a membrane deposit undoubtedly forms during CFM. The deposit is known to be composed of particles from the finer size ranges (Milisic et al. (1986b); Fischer et al. (1986); Murkes (1986)) and may be considered to constitute a 'dynamic membrane' on the surface of the microporous filter medium.

The dynamic membrane acts rather like a filter aid and is capable of retaining very fine particles. Thus, possibly the only application of classical filtration theory to CFM (whether electrically assisted or otherwise) is the calculation of the average specific resistance of the membrane deposit which forms during the first few moments of separation. However, it should be noted that this requires the initial portion of the curve to be approximated by a straight line and can at best only provide a rough estimate. Observations of the rate at which a filter medium blinds during filtration have indicated the existence of four fundamental types of filtration mechanisms (Hermans (1935)). The mechanisms describe methods by which particulate fouling of a membrane pore can occur and it is possible to express each mechanism in terms of an equation that relates the flux depletion during filtration to the properties of the process suspension (Hermia (1982)). The equations have been applied to the data shown in Figures 1 and 2 and the results are shown in Figures 4 and 5. 
It is evident from Figure 4 that the flux depletion obtained for the CFM of a dilute china clay suspension (no electric field applied) cannot be adequately described by one particulate blocking mechanism alone. Rather, the particulate component of the membrane fouling probably occurs by a combination of two or more mechanisms with the overall fouling process possibly complicated by other factors such as chemical fouling. With an increased suspension concentration the effects of particle fouling appear to be increased. Figure 5 shows, for the given experimental conditions, that the standard blocking mechanism can offer some description of the flux depletion, albeit rather poorly. Moreover, the graph suggests that at a high solids concentration the membrane fouling processes are likely to be dominated by particle deposition at the membrane surface (i.e. particle polarisation). Although the blocking laws can be used to describe crossflow filtration under certain conditions, an attempt to modify them to account for the presence of external fields such as electric or sonic fields may introduce factors which are difficult to quantify (cf. Henry et al. (1977)). It is perhaps not surprising that the filtration blocking law better describes the CFM of a more concentrated suspension, as it is this which the laws were intended to describe.

\section{b) Ultrafiltration Theory and Microfiltration}

It has been widely reported that during ultrafiltration the permeate flux through the membrane is related to the resistance of the membrane deposit or gel-layer (Bertera (1984); Fane (1986); Kristensen (1986)). The gel-layer forms as a consequence of the combination of the action of the crossflowing stream and the diffusional migration of colloids and/or macromolecules toward the membrane. However, during CFM gel formation is rarely observed because of the presence of relatively large particles or agglomerates in the process suspension. Thus, it is difficult to apply UF theories to CFM, at least not without applying extensive and possibly unrealistic restrictions.

Further, due to the different physical processes involved in UF and CFM it is unlikely that some of the more rigorous UF models (Fane (1986); Le et al. (1983)) could provide an adequate description of CFM. Hence, UF theory has not been used as a method of analysis.

\section{c) Other Crossflow Theories and Microfiltration}

The theories propounded for cake filtration and UF can be applied to CFM in a fairly limited sense. The addition of an external electric field compounds the problems associated with their application. However, several other models have been proposed in the literature for analysing crossflow data and some of these are discussed here. Mikhlin et al. (1982) have derived a mathematical model which is purported to parallel that used in sedimentation analysis, and this can be used to estimate the average specific resistance of the membrane deposit that forms during crossflow filtration. The degree of flux depletion during filtration was considered to be proportional to the rate at which particles accumulate at the membrane surface. Mikhlin included factors to account for both the deposition and removal of particles from the membrane surface and gave the following expression to describe the flux decline:

$$
\left(\frac{v_{w i}}{v_{w \infty}}\right)^{2} \log _{e}\left(\frac{1-v_{w \infty} / v_{w i}}{1-v_{w \infty} / v_{w}}\right)-\frac{v_{w i}}{v_{w \infty}}\left(\frac{v_{w i}}{v_{w}}-1\right)=\frac{\alpha_{a v} \mu c}{\Delta p} v_{w}^{2} t
$$

where $v_{w i}$ is the initial permeate flux $(t=0 \mathrm{~s}), v_{w^{\infty}}$ the permeate flux at equilibrium and $v_{w}$ the permeate flux.

Figure 6 shows how this equation can be used to represent the flux decline for the crossflow filtration of a dilute anatase suspension. The theoretical curve was fitted to the experimental curve by minimising the variance between the two. The theory adequately described the experimental flux depletion both with and without an imposed electric field and facilitated the calculation of the average specific resistance of the membrane deposit. The relatively small reduction in the deposit resistance when the field was applied contrasts sharply with results obtained for deadend electrofiltration and is indicative of the minimal 'cake' formation during CFM. This implies that 
membrane electroosmosis may provide a significant contribution to the permeate flux in electrofiltration. Similar results were found when other crossflow data were analysed, and overall Mikhlin's model was found to be a versatile method of analysis.

Lee et al. (1980) have produced a model of electrically assisted CFM which proposed that, when the electric field strength is greater than a critical value, a clear boundary layer is formed adjacent to the separating surface. (In this way a clear filtrate may be produced without the use of a semipermeable membrane!) Although Lee's analysis is interesting, the theory is difficult to apply to an arbitrary set of experimental data. The formation of a clear boundary layer is only well defined when a critical field strength is achieved and Lee's proposal that a clear filtrate can only be produced at or above this field strength is not applicable when a membrane is used to achieve separation. The formation of the clear boundary layer is essentially hypothetical under these circumstances, and as such Lee's model should not be used to describe the flux performance during crossflow filtration.

Another model was proposed by Zhenovatyi (1975) who produced a semi-empirical correlation that related permeate flux to crossflow velocity for the crossflow filtration of a suspension involving no 'cake' formation.

$v_{w}=4.6 \times 10^{-7}\left(\frac{d}{L}\right)^{0.41} \mathrm{Eu}^{0.42} \operatorname{Re}^{0.88}\left(\frac{K \Delta p}{\mu t_{m}}\right)^{-0.4}$

where $L$ is the length of the membrane, $d$ the outer diameter of the tubular membrane, Eu the Euler number of the crossflow stream, Re the Reynolds number of the crossflow stream, $K$ the membrane permeability and $t$ the membrane thickness. Some of the experimental data obtained from the crossflow filter were analysed using the correlation and the results are shown in Table 1. The poor comparison obtained between the theoretical and experimental fluxes highlights some of the problems associated with this model. The results shown here and elsewhere (Rushton et al.) indicate that the model may only be applicable to the filter and filtration conditions used by Zhenovati to obtain the original correlation.

The final model discussed in this section is one that has been previously proposed by the authors (Wakeman and Tarleton (1986a); (1987)). The method relies on the calculation of the trajectories of particles introduced at one end of a crossflow filter. The solution of the relevant equations of motion for each particle allows an estimation of the degree of particulate membrane fouling that occurs during filtration. Figure 7 shows the calculated trajectories for particles fed at two different positions. With no electric field applied, those fed at a position close to the porous surface are rapidly pulled onto the surface. As the field gradient increases, so these particles remain in suspension for a greater distance. Particles fed in halfway across the annular section, however, showed little tendency to approach the surface, even when no field was applied. Although the results are specific to the conditions quoted, it appears that only particles fed close to the surface of the filter will create a fouling problem. If this is the case then careful design of a crossflow filter is required, particularly in the immediate vicinity of the membrane surface. This suggests that not only should the shearing action of the crossflow stream be maximised here, perhaps by promoting a certain degree of turbulence, but also any applied external field should also be optimised within this region.

\section{DISCUSSION}

The experimental data presented in this paper demonstrate the effectiveness of crossflow microfiltration, particularly when the process is assisted by the presence of an external electric field. The data were obtained over a range of operating conditions and the flux depletion was 
dependent on many contributory factors. The membrane fouling processes which cause flux depletion are numerous and some of these are shown schematically in Figure 8.

Without the addition of any external fields, the flux performance during CFM is probably controlled by particle accumulation within the vicinity of the membrane surface. Experimental data have shown that this 'particle polarisation' is ostensibly governed by the velocity of the crossflowing stream, the permeate flux (related to the filtration pressure) and the concentration of the process suspension. Particle deposition was found to be extensive when suspensions with volume fractions of solids greater than $0.1 \%$ were filtered, and this caused rapid fouling of the membrane and subsequently low separation rates. The mathematical analysis of crossflow filtration under these conditions confirmed that particle polarisation is a predominant cause of membrane fouling, and showed that particulate fouling probably occurs primarily by a mechanism whereby the membrane pores are progressively blocked internally. The pore blocking is likely to be caused by particle 'fines' which accumulate throughout the initial period of filtration and result in the formation of a secondary or dynamic membrane on the filtering membrane. This situation may be further aggravated if the process suspension is predominantly composed of colloidal sized particles where surface effects are important. With a more dilute suspension the particulate component of membrane fouling is still probably extensive, however, the mechanism by which it occurs may be influenced to a greater extent by complete blocking of membrane pores.

The lack of formation of a true filter cake during CFM can be attributed to the scouring action of the crossflow stream. It is interesting to note, therefore, that increasing the velocity of the crossflow stream often produces only small reductions in membrane fouling and may sometimes even result in increased fouling (Fischer et al. (1986)). Such results appear to corroborate the idea that internal blocking of membrane pores is a primary fouling mechanism during CFM, although to some extent particle classification in the boundary layer adjacent to the membrane may contribute.

When crossflow microfiltration is electrically assisted, several electrokinetic effects are introduced which modify the membrane fouling process. Both electrophoresis and electroosmosis were found to reduce membrane fouling, and in some cases almost completely eliminate it. However, it seems unlikely that this ideal situation can be regularly repeated in practice. If separation is achieved at, or close to, a maximum rate, the action of an imposed electric field will probably not totally prevent the formation of a membrane deposit. Rather, a deposit will form which exhibits a negligible specific resistance.

Although particle polarisation is probably the major cause of fouling in CFM other factors should also be considered. In recent years membrane design has been the subject of much research and development and this has led to the production of membranes more tailored to the requirements of crossflow filtration. Whilst the use of asymmetric membranes with narrow pore size distributions has become almost universal, the choice of membrane material is forever increasing. It is widely recognised that membrane fouling in RO and UF can be reduced if the membrane is chosen carefully, particularly if the particulates in the feed suspension exhibit a high surface charge (Wakeman (1982)). There is some evidence to suggest, however, that the membrane material choice in CFM is less critical, provided all other factors are equal (Tarleton (1986); Fischer et al. (1986)). This does not necessarily imply that membrane choice is arbitrary. If a large proportion of colloidal material is present in the feed or corrosive suspensions are used, then membrane choice may still be important. The separation of corrosive materials by CFM may also introduce an element of chemical membrane fouling and this is likely to be increased if an electric field is used to assist the process. Here, the products of electrolysis can not only contaminate the membrane but also alter such parameters as the $\mathrm{pH}$ of the process suspension. If both the particulate and chemical constituents of membrane fouling are to be controlled it is likely that not only is appropriate design of the filter internals necessary, but careful control of the suspension flow characteristics is also essential, as is the handling and flow of any gases which are generated. 
For this electrical technique to become industrially viable the power consumption needs to be quite small. Electric power consumption is mainly dependent on two factors, the conductivity of the process suspension and the geometric design of the separator (Wakeman and Tarleton (1987)). In the crossflow unit described above the applied voltage was held constant during experiments, whilst the current was variable. Typical correlations between the permeate volume collected and the electric power consumption with field strength as a parameter are shown on Figure 9(a) for a china clay slurry. These are fitted by the empirical equation

$V=a W_{p}^{n}$

where $a$ and $n$ are experimental constants. The value of $n$ approaches unity as the field strength increases $-n$ is probably also dependent on filter configuration. A larger value of $a$ indicates less power consumption for the collection of any specified permeate volume. Figure 9(b) shows the power consumption as a function of time with field strength as a parameter. These plotted data have a slope of unity, and, as might be expected,

$\frac{d W_{p}}{d t}=($ field strength $)($ current drawn $)($ electrode separation $)$

i.e. the power consumption could be lowered by using closer spaced electrodes. This would also enable lower voltages to be used and reduce the tendency towards such undesirable effects as electrolysis of the process liquor.

\section{CONCLUSIONS}

It seems evident that fouling in crossflow filtration is likely to remain a problem for many years to come, and may possibly never be completely eradicated. Although the crossflow technique has shown considerable promise over the past years, the apparent complexity of the mechanisms that cause flux decline has largely prevented progress being made toward modelling the problem. Many of the mechanisms do not appear to be completely understood, and as a result use of existing models are limited to the analysis of experimental data rather than providing an understanding of the phenomenon or a method by which filter performance can be predicted. If the crossflow technique is to be improved it is essential that a better understanding of the mechanisms which cause fouling is obtained. For example, it would seem pertinent to investigate the behaviour of macromolecular and particulate material which move close to the membrane surface during crossflow, in this way improved filter design may be facilitated. In order to prevent particulate deposition completely it would probably be necessary to eliminate all particulates from the region immediately adjacent to the membrane. Whilst this situation is likely to be difficult to achieve in practice, the use of an applied electric field has been shown to reduce the rate of flux decline. With further development it seems probable that this technique will provide an alternative, cost effective method of separating difficult to filter materials.

\section{NOMENCLATURE}

$\begin{array}{ll}A & \text { filtration area }\left(\mathrm{m}^{2}\right) \\ c & \text { mass of solid/unit volume filtrate }\left(\mathrm{kg} \mathrm{m}^{-3}\right) \\ d & \text { diameter of tubular membrane }(\mathrm{m}) \\ \mathrm{Eu} & \text { Euler number of the crossflow stream } \\ K & \text { membrane permeability }\left(\mathrm{m}^{2}\right) \\ L & \text { length of membrane }(\mathrm{m}) \\ \Delta p & \text { hydraulic pressure differential }\left(\mathrm{N} \mathrm{m}^{-2}\right) \\ r_{i} & \text { inner radius of tubular annulus }(\mathrm{m})\end{array}$


$r_{o} \quad$ outer radius of tubular annulus $(\mathrm{m})$

$R_{m} \quad$ filter medium resistance $\left(\mathrm{m}^{-1}\right)$

$\mathrm{Re}$ Reynolds number of the crossflow stream

$t$ time (s)

$t_{m} \quad$ membrane thickness $(\mathrm{m})$

$v_{w} \quad$ permeate flux $\left(\mathrm{m}^{3} \mathrm{~m}^{-2} \mathrm{~s}^{-1}\right)$

$v_{w i} \quad$ initial permeate flux $\left(\mathrm{m}^{3} \mathrm{~m}^{-2} \mathrm{~s}^{-1}\right)$

$v_{w^{\infty}} \quad$ permeate flux at equilibrium $\left(\mathrm{m}^{3} \mathrm{~m}^{-2} \mathrm{~s}^{-1}\right)$

$V \quad$ volume of filtrate $\left(\mathrm{m}^{3}\right)$ or $\left(\mathrm{m}^{3} \mathrm{~m}^{-2}\right)$

$W_{p} \quad$ electric power $(\mathrm{kWh})$ or $\left(\mathrm{kWh} \mathrm{m}^{-2}\right)$

Greek symbols

$\alpha_{a v} \quad$ average specific resistance of filter cake $\left(\mathrm{m} \mathrm{kg}^{-1}\right)$

$\mu \quad$ dynamic viscosity $\left(\mathrm{N} \mathrm{s} \mathrm{m}^{-2}\right)$

\section{REFERENCES}

Bertera R.G., Metcalfe M.G., and Steven J.H., 1984, Development studies in crossflow microfiltration, The Chemical Engineer, June,10-14.

Dolle L., 1987, Electromagnetic filtration process development, Proc. Filtech Conference, pp.44-52, Utrecht, Holland.

Fane A.G., 1986, Ultrafiltration: Factors influencing flux and rejection, in Progress in Filtration and Separation, Vol. 4, Wakeman R.J. (Ed.), Elsevier, Amsterdam.

Fischer E. and Raasch J., 1986, Model tests of particle deposition at the filter medium in crossflow filtration, Proc. $4^{\text {th }}$ World Filtration Congress, Ostend, Belgium, April.

Henry J.D., Lawler L.F. and Alex Kuo C.H., 1977, A solid/ liquid separation process based on crossflow and electrofiltration, A/ChEJ, 23, 851.

Hermans P.H. and Bredee H.L., 1935, Rec. Trav. Chim. des Pays Bas, 54, 680.

Hermia J., 1982, Constant pressure blocking filtration laws - Application to power-law nonNewtonian fluids, Trans. IChemE, 60, 183.

Kristensen S., 1986, Ultrafiltration and microfiltration in the biotechnological industry, Proc. $4^{\text {th }}$ World Filtration Congress, Ostend, Belgium, April.

Le M.S. and Howell J.A., 1983, Model for the effects of adsorbents and cleaners on ultrafiltration membrane structure, Chem. Eng. Res. Dev., 61, 191-197.

Lee C.H., Gidaspow D. and Wasan D.T., 1980, Crossflow electrofilter for non-aqueous slurries, Ind. Eng. Chem. Fundam., 19, 166-175.

Mikhlin J.A., Weber M.E. and Turkson A.K., 1982, Electrically-aided axial filtration, J. Separ. Proc. Technol., 3, 16-24.

Milisic V. and Ben Aim R., 1986a, Developing a better understanding of crossflow microfiltration, Filtration and Separation, Jan/Feb, 28-30. 
Milisic V. and Bersillon, J.L., 1986b, Antifouling techniques in crossflow microfiltration, Proc. $4^{\text {th }}$ World Filtration Congress, Ostend, Belgium, April.

Muralidhara H.S. (Ed.), 1986, Advances in Solid-Liquid Separation, Battelle Press, Ohio.

Murkes J., 1986, Crossflow filtration of emulsions combined with coalescing, Proc. $4^{\text {th }}$ World Filtration Congress, Ostend, Belgium, April.

Rushton A. and Aziz R., Private communication.

Tarleton E.S., 1986, PhD Thesis, University of Exeter.

Turkson A.K., 1987, Electrofiltration of bovine serum albumin with rotating dynamic membranes, Proc. Filtech Conference, pp.294-301, Utrecht, Holland.

Wakeman R.J., 1986, Electrofiltration: Microfiltration plus electrophoresis, The Chemical Engineer, June, 65-70.

Wakeman R.J. and Tarleton E.S., 1986a, Modelling crossflow electro- and micro- filtrations, Proc. $4^{\text {th }}$ World Filtration Congress, Ostend, Belgium, April.

Wakeman R.J. and Tarleton E.S., 1986b, Experiments using electricity to prevent fouling membrane filtration, Filtration and Separation, May/June, 174-176.

Wakeman R.J. and Tarleton E.S., 1987, Membrane fouling prevention in crossflow microfiltration by the use of electric fields, Chem. Eng. Sci., 42(4), 829-842.

Zhenovatyi A.I., 1975, Basic principles of filtration of suspensions in flow without formation of deposit, J. Applied Chem. USSR, 48(2 pt 1), 335-339. 


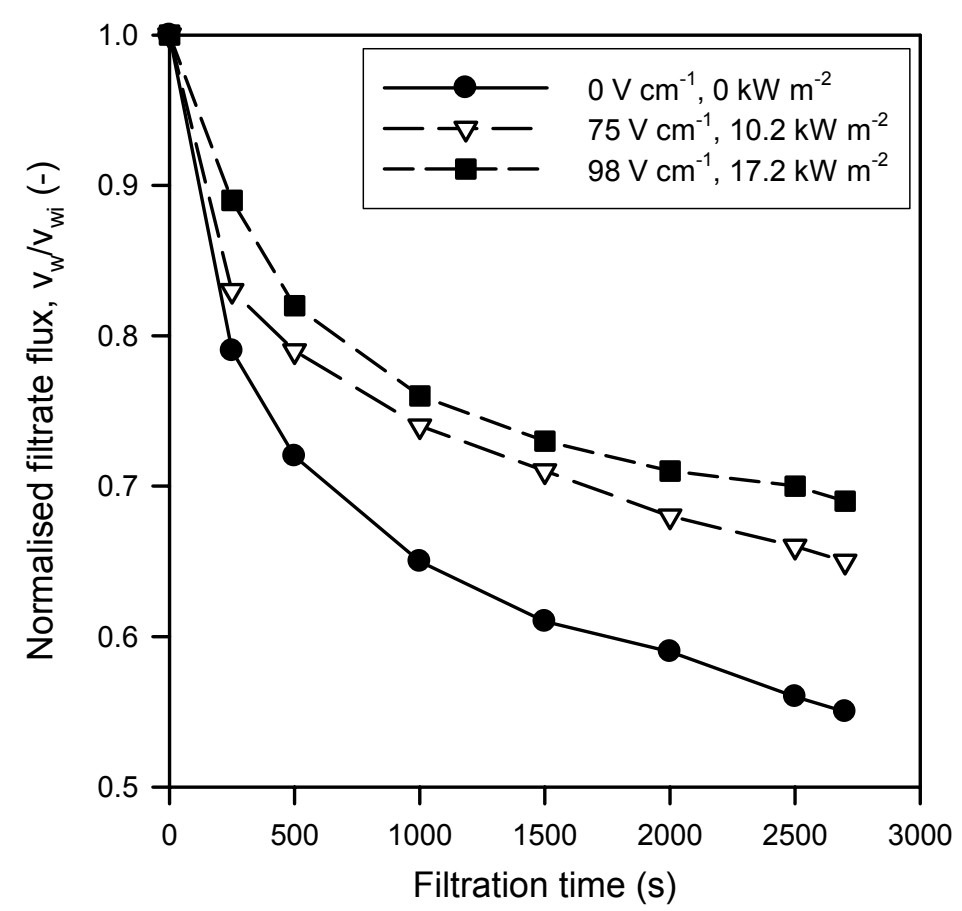

Figure 1: Crossflow filtration of $0.0039 \% \mathrm{v} / \mathrm{v}$ china clay in water. Crossflow velocity $=0.9 \mathrm{~m} \mathrm{~s}^{-1}$; Initial filtration rate $=2.91 \mathrm{~m}^{3} \mathrm{~m}^{-2} \mathrm{~h}^{-1}$; Suspension conductivity $=1.426 \times 10^{-4} \mathrm{ohm}^{-1} \mathrm{~cm}^{-1}$; Membrane type $=$ pleated polypropylene; Electrode separation $=4 \mathrm{~cm}$.

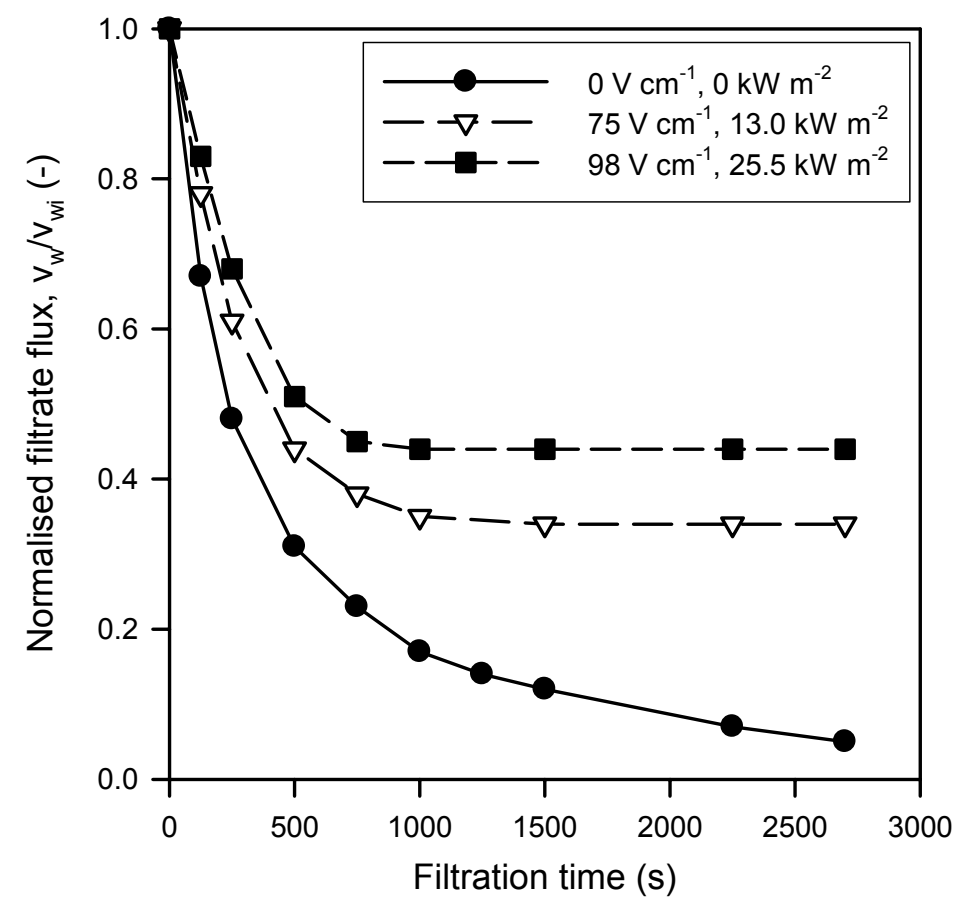

Figure 2: Crossflow filtration of $0.39 \% \mathrm{v} / \mathrm{v}$ china clay in water. Crossflow velocity $=0.9 \mathrm{~m} \mathrm{~s}^{-1}$; Initial filtration rate $=0.97 \mathrm{~m}^{3} \mathrm{~m}^{-2} \mathrm{~h}^{-1}$; Suspension conductivity $=1.439 \times 10^{-4} \mathrm{ohm}^{-1} \mathrm{~cm}^{-1}$; Membrane type $=$ pleated polypropylene; Electrode separation $=4 \mathrm{~cm}$. 


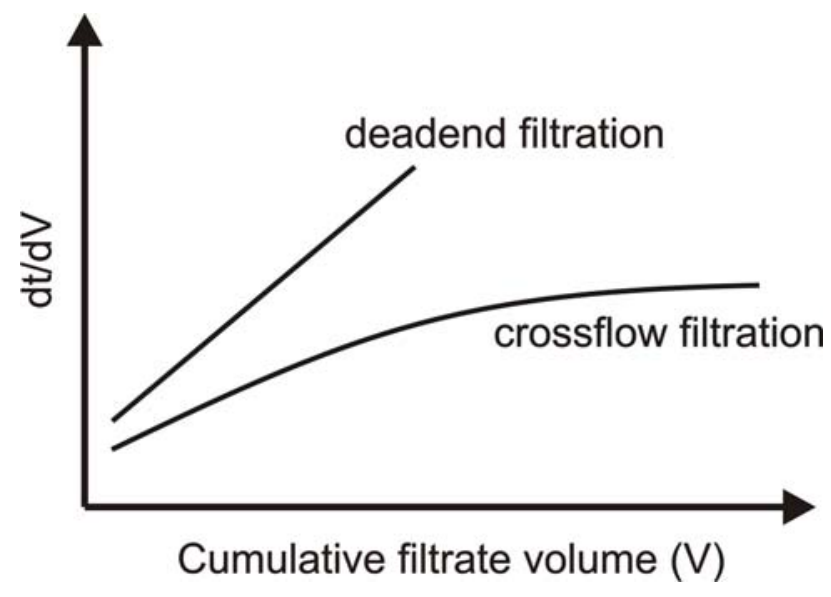

Figure 3: Typical dt/dV vs. V plots for deadend and crossflow filtration.

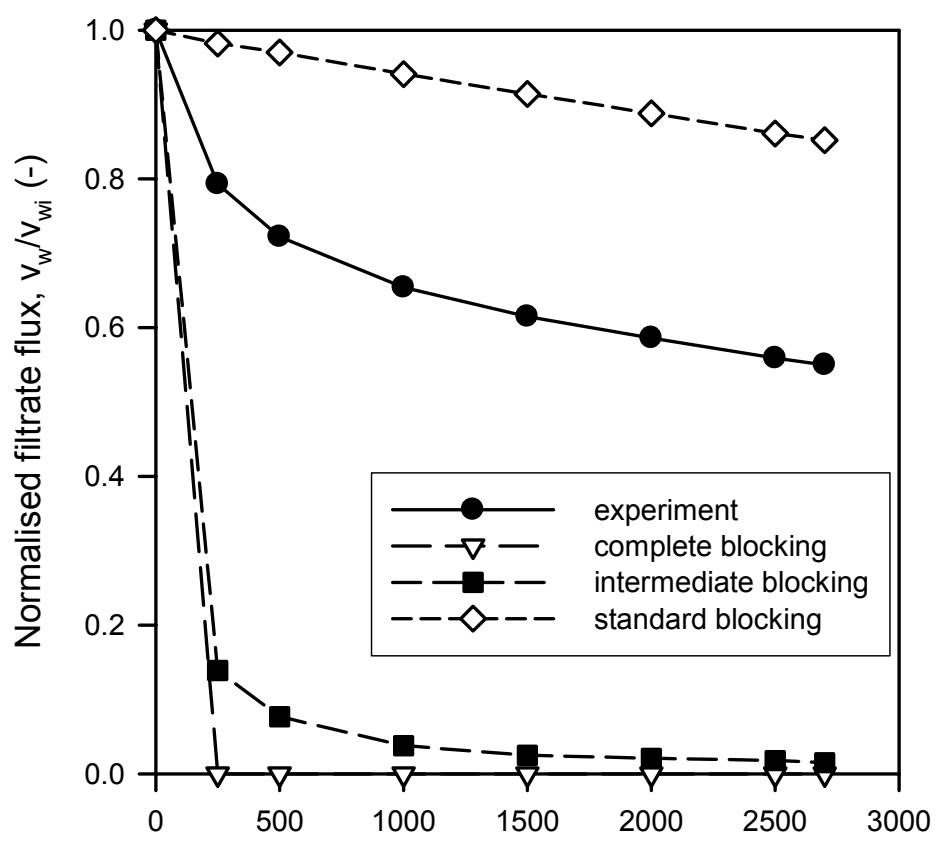

Filtration time (s)

Figure 4: Filtration blocking laws applied to crossflow filtration of $0.0039 \% \mathrm{v} / \mathrm{v}$ china clay in water. 


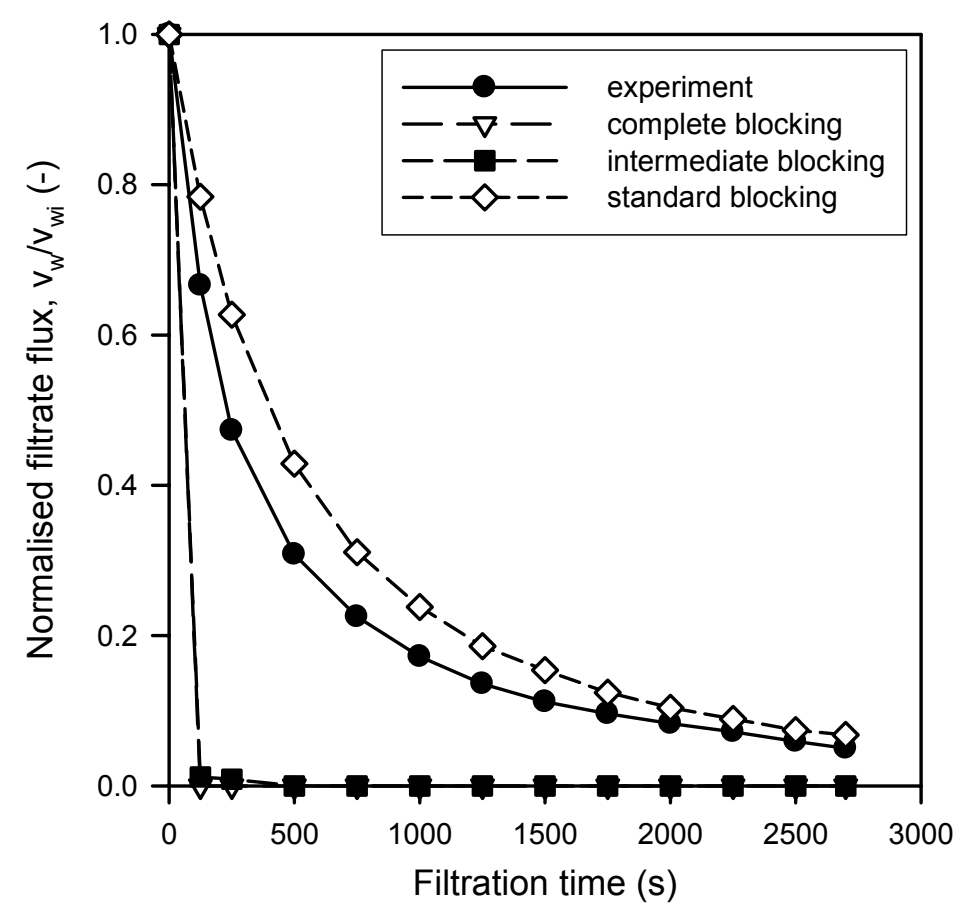

Figure 5: Filtration blocking laws applied to crossflow filtration of $0.39 \% \mathrm{v} / \mathrm{v}$ china clay in water.

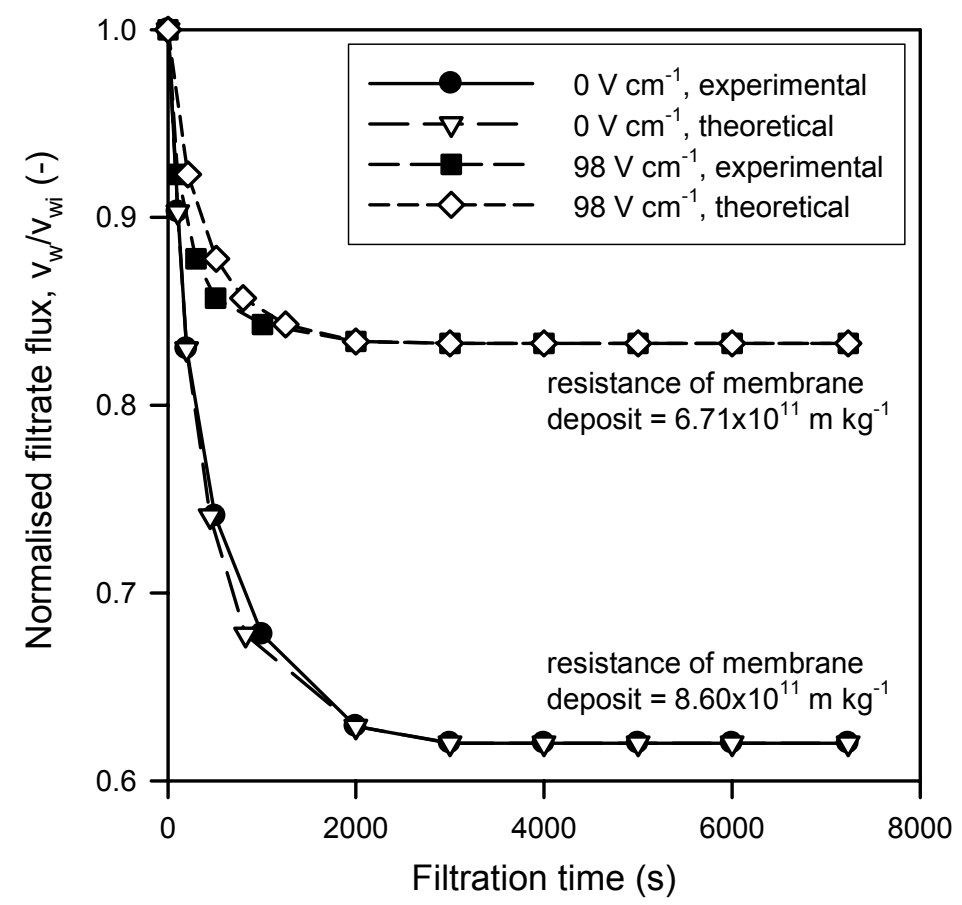

Figure 6: Comparison between experimental and theoretical flux decline using Mikhlin's model. Solids $=$ anatase; Suspension concentration $=0.0049 \% \mathrm{v} / \mathrm{v}$; Initial filtration rate $=2.63 \mathrm{~m}^{3} \mathrm{~m}^{-2} \mathrm{~h}^{-1}$. 


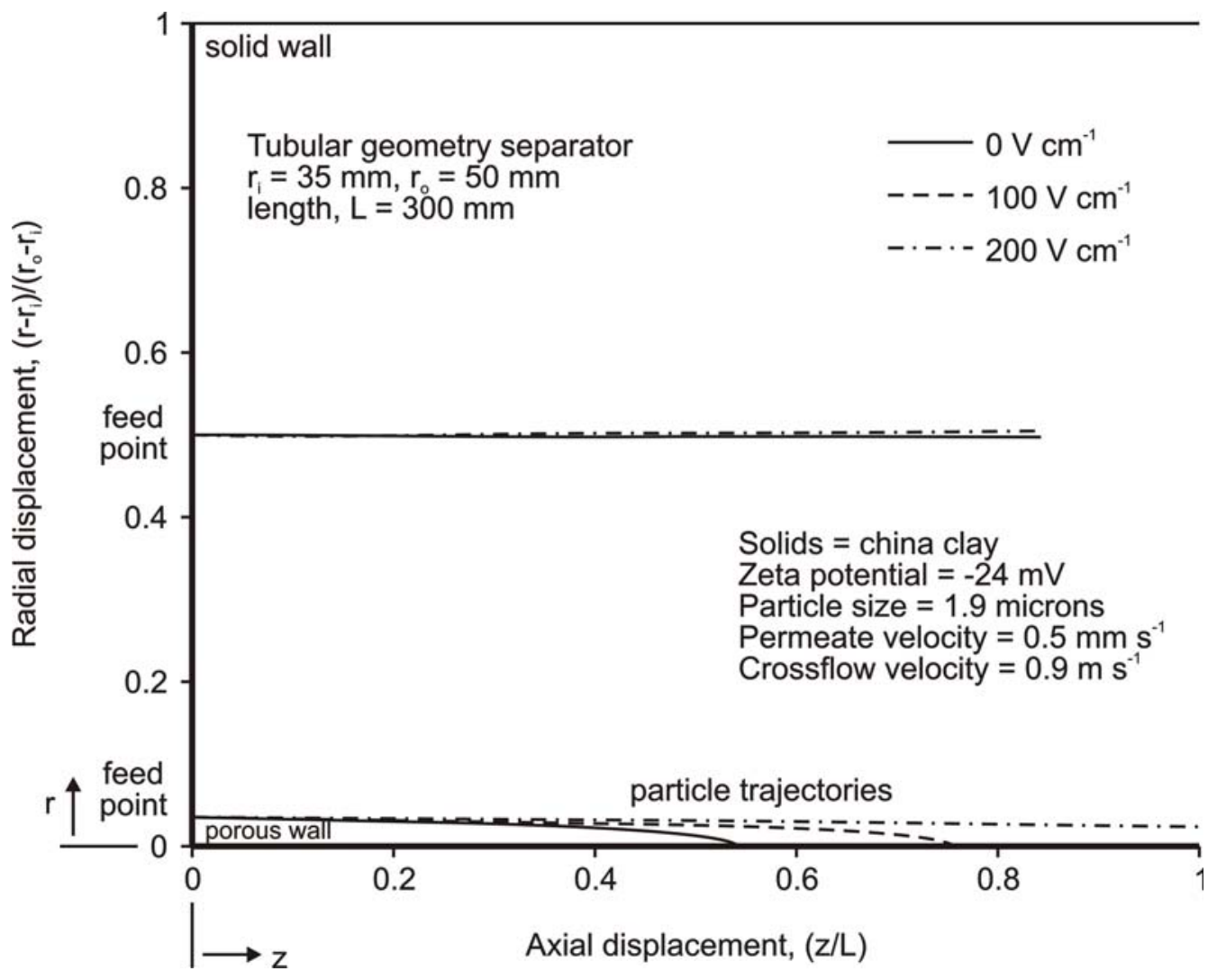

Figure 7: Effect of electric field strength on particle trajectory.

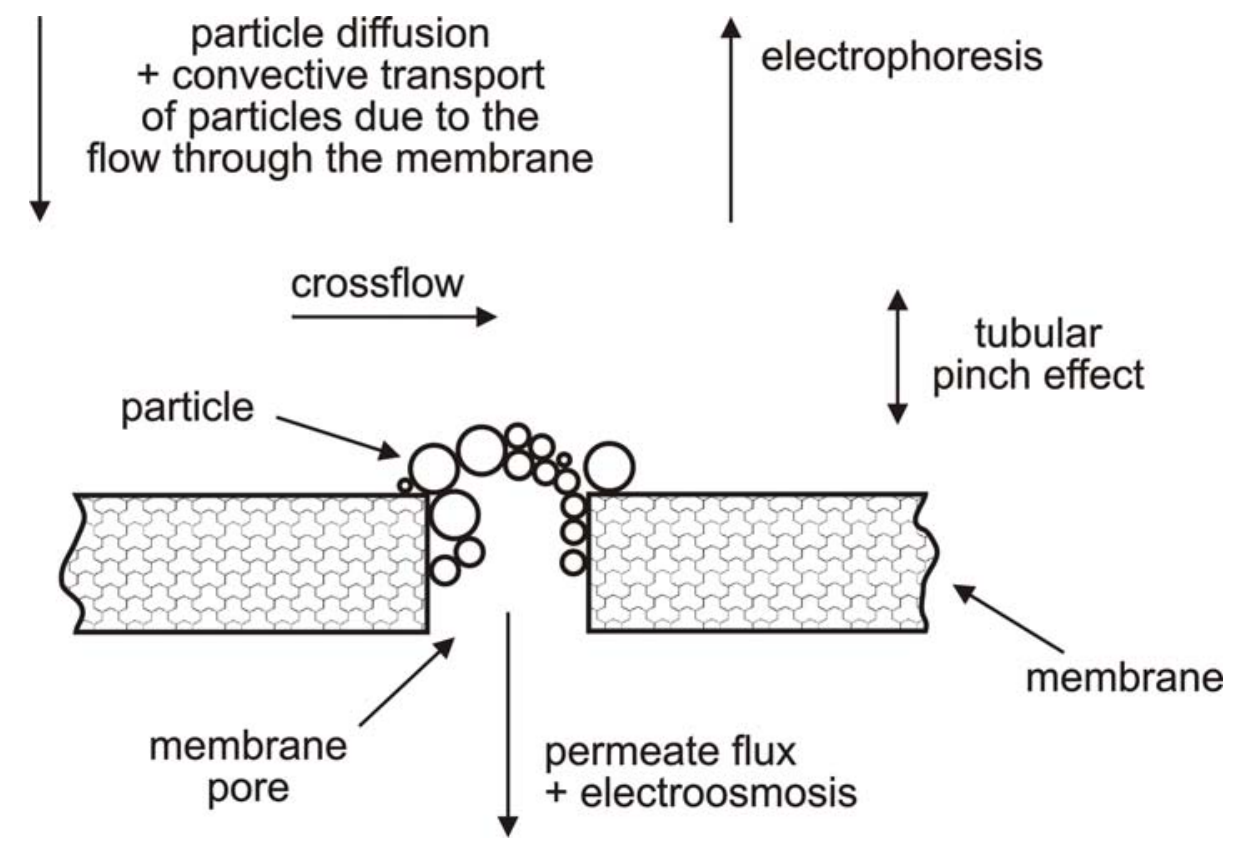

Figure 8: Contributions to membrane fouling during crossflow filtration. 


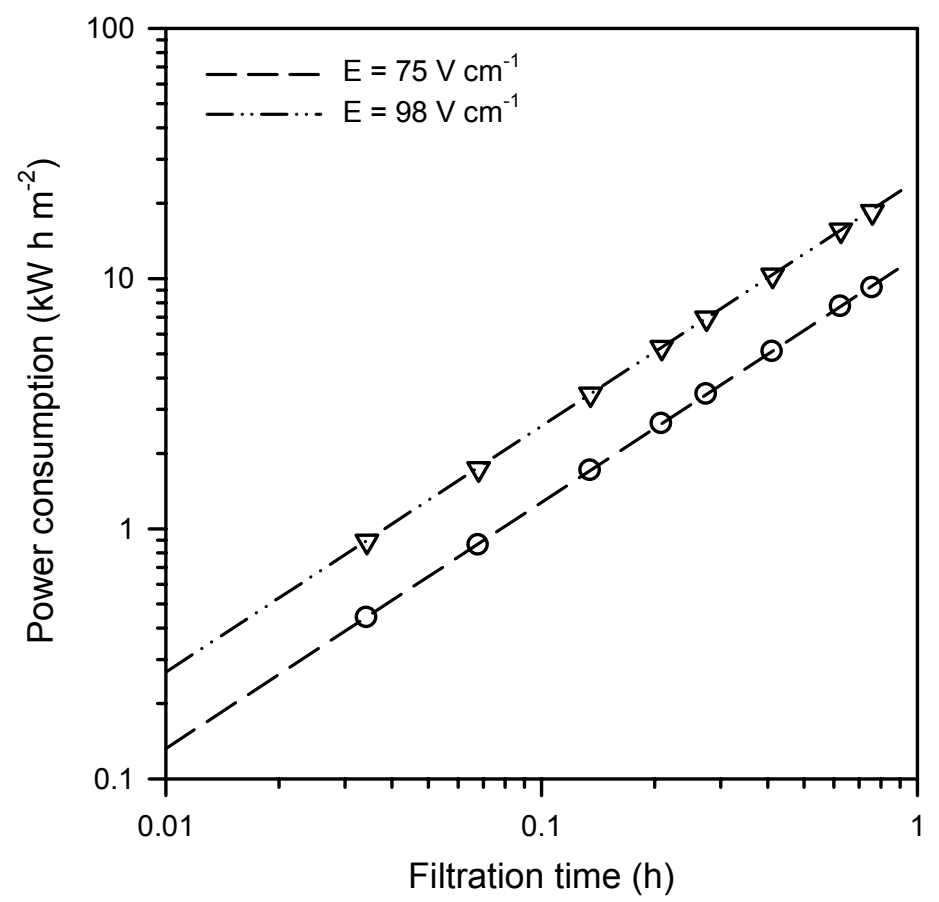

Figure 9a: Power consumption for data on Figure 2.

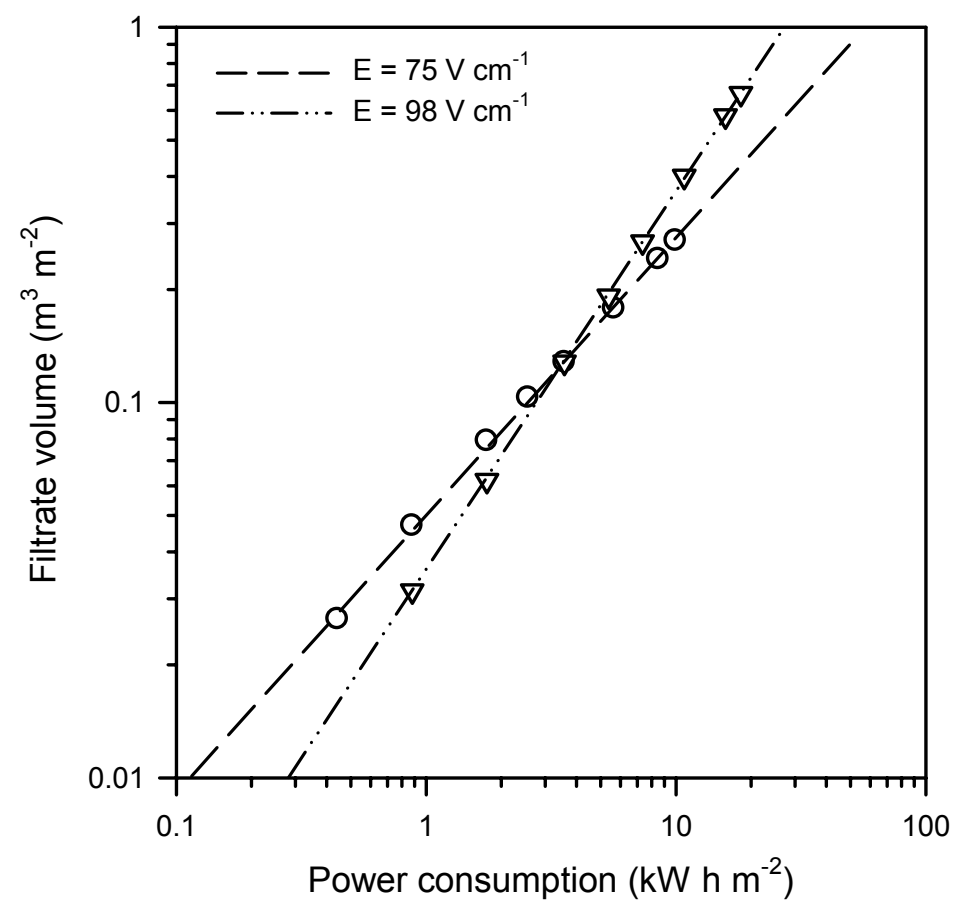

Figure 9b: Permeate volume vs. power consumption for data on Figure 2. 
Solids: china clay

\begin{tabular}{|c|c|c|c|c|}
\hline \multicolumn{5}{|c|}{ Solids: china clay } \\
\hline $\begin{array}{c}\text { Experiment } \\
\text { number }\end{array}$ & $\begin{array}{c}\text { Suspension } \\
\text { conc. }(\% \mathrm{v} / \mathrm{v})\end{array}$ & $\begin{array}{c}\text { Permeate flux at } \\
t=0 \mathrm{~s}\left(\mathrm{~m}^{3} \mathrm{~m}^{-2} \mathrm{~s}^{-1}\right)\end{array}$ & $\begin{array}{c}\text { Permeate flux at } \\
t=2700 \mathrm{~s}\left(\mathrm{~m}^{3} \mathrm{~m}^{-2} \mathrm{~s}^{-1}\right)\end{array}$ & $\begin{array}{c}\text { Flux predicted by } \\
\text { correlation }\left(\mathrm{m}^{3} \mathrm{~m}^{-2} \mathrm{~s}^{-1}\right)\end{array}$ \\
\hline 54 & 0.0013 & $5.61 \times 10^{-4}$ & $3.31 \times 10^{-4}$ & $9.92 \times 10^{-3}$ \\
60 & 0.019 & $3.81 \times 10^{-4}$ & $1.79 \times 10^{-4}$ & $9.92 \times 10^{-3}$ \\
68 & 0.19 & $1.33 \times 10^{-4}$ & $2.80 \times 10^{-5}$ & $9.90 \times 10^{-3}$ \\
77 & 0.97 & $8.06 \times 10^{-5}$ & $5.64 \times 10^{-6}$ & $9.85 \times 10^{-3}$ \\
\hline
\end{tabular}

Table 1: Comparison of crossflow experiment fluxes and the fluxes predicted using Zhenovatyi's correlation. 